\title{
OSCILLATION OF THE MAGNETIC FIELD IN AN ACTIVE REGION
}

\author{
T. HORN AND J. STAUDE \\ Astrophysikalisches Institut Potsdam \\ Telegrafenberg A27; D-14473 Potsdam - Germany
}

Observations. The Vacuum Tower Telescope (VTT) at Tenerife has been used together with a polarimeter in front of a two-dimensional imaging spectrometer with a Fabry-Perot interferometer. The spectrometer has been described by Bendlin et al. (1992) and Bendlin et al. (1995), the remaining instrumentation, calibration, and data reduction by Horn et al. (1996). Some parameters of the setup were: $384 \times 286$ pixel CCD-camera ( 0.2 " per pixel); resolution for the images better than 0.8 "; filtergrams in the photospheric spectral line Fe I $6173.4 \AA$ with $\Delta \lambda=10.9 \mathrm{~m} \AA ; 45$ filtergrams for each scan; 127 scans within $114 \mathrm{~min} . \Delta t=54 \mathrm{~s}$. White-light images from the same field of view were taken strictly simultaneously to all narrow-band pictures to correct for image blurring and motion.

The present observations were obtained on July 20, 1994, and were focused on the main spot of the active region NOAA $7757,30^{\circ} \mathrm{NE}$ from the disk center.

Results. From the $(I+V)$ and $(I-V)$ images the Doppler shifts (velocity $v$ ) and the Zeeman splittings of the observed line were derived by fitting a polynomial to the line cores of the $\sigma$-components. The measured splittings are influenced by the total field strength $B$ and to a minor extent by the angle of inclination between the line-of-sight and the vector $B$, if the splitting is incomplete. The maps show the power of $v$ and $\delta B$ in two bands of frequency $\nu$ (around $P \approx 3 \mathrm{~min}$ and $5 \mathrm{~min}$, respectively), moreover, the coherence and phase difference $\Delta \Phi$ between both types of oscillations are given. The power spectra of $v(t)$ in the umbra show the known features of strong power in bands of periods around $3 \mathrm{~min}$ (strengthened) and $5 \mathrm{~min}$ (weakened with respect to the quiet Sun). The data show significant power of oscillations of $\delta B(t)$ as well, which is concentrated in the same frequency bands as the power of $\delta v(t)$.

To exclude a possible influence of the jitter of the telescope the intensity fluctuation of a small area at the umbra-penumbra boundary has been checked: significant power is observed at frequencies $\nu<1.5 \mathrm{mHz}$ only. An inspection of the observed umbral contrast $\phi$ shows a large amount of stray light, but it is very stable in time: the power density of the fluctuations of $\phi$ is very weak and equally distributed over all $\nu$.

The spatial distribution of the power of $v(t)$ and $\delta B(t)$ is inhomogeneous across the umbra and shows marked spatial fine structures. Maximum power is measured in those parts of the umbra (close to the umbra-penumbra boundary) where we are looking along the lines of force of $\mathbf{B}$, thus demonstrating the longitudinal character of the oscillations with respect to the direction of $\mathbf{B}$. In the same parts of the umbra 

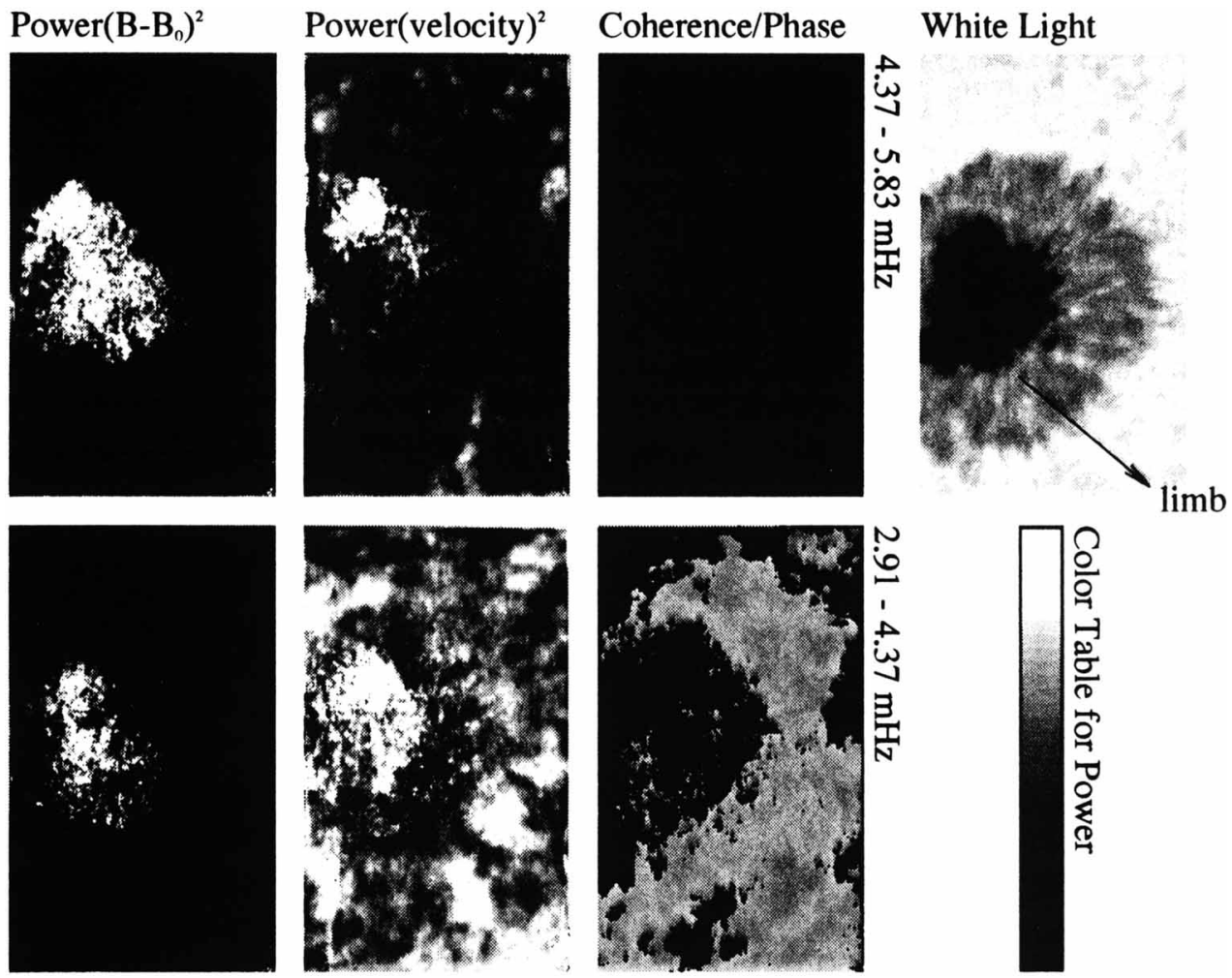

Figure 1. Spatial distribution of power of the oscillations of $B(t)$ (left), $v(t)$ and phase difference $\Delta \Phi$ (middle) for two selected $\nu$ bands; the right column shows the direction towards the limb, the power gray scale (maximum power is brightest), and an $I_{c}$ picture of the same region. The gray coding for the $\Delta \Phi$ map is: black for a coherence $<0.85$, elsewhere gray for $\Delta \Phi \approx \pi / 2$ and white for $\Delta \Phi \approx 0$

we found also the largest correlation and $\Delta \Phi \approx 0$ between both types of oscillations, in the 3-min band in particular. The penumbral regions show rather an quadrature in the 5-min band.

\section{References}

Balthasar, H., Schleicher, H., Bendlin, C.,and Volkmer, R. (1996) Astron. Astrophys. 315, 603

Bendlin, C., and Volkmer, R. (1995) Astron. Astrophys. 112, 371

Bendlin, C., Volkmer, R., and Kneer, F. (1992) Astron. Astrophys. 257, 817

Horn, T., Hofmann, A., and Balthasar, H. (1996) Solar Phys. 164, 321

Horn, T., Hofmann, A., and Landgraf, V. (1997) Solar Phys. 172, 69 